Draft Version August 3, 2021

Preprint typeset using $\mathrm{LAT}_{\mathrm{E}} \mathrm{X}$ style AASTeX6 v. 1.0

\title{
FLARES ON A-TYPE STARS: EVIDENCE FOR HEATING OF SOLAR CORONA BY NANOFLARES?
}

\author{
Michal Švanda \\ Astronomical Institute, Charles University in Prague, Faculty of Mathematics and Physics, V Holešovičkách 2, CZ-18000 Prague 8, \\ Czech Republic \\ and \\ Astronomical Institute (v. v. i.), Czech Academy of Sciences, Fričova 298, CZ-25165 Ondřejov, Czech Republic \\ MARIAN KARLICKÝ \\ Astronomical Institute (v. v. i.), Czech Academy of Sciences, Fričova 298, CZ-25165 Ondřejov, Czech Republic
}

\begin{abstract}
We analyzed the occurrence rates of flares on stars of spectral types K, G, F, and A, observed by Kepler. We found that the histogram of occurrence frequencies of stellar flares is systematically shifted towards a high-energy tail for A-type stars compared to stars of cooler spectral types. We extrapolated the fitted power laws towards flares with smaller energies (nanoflares) and made estimates for total energy flux to stellar atmospheres by flares. We found that for A-type stars the total energy flux density was at least 4-times smaller than for G-stars. We speculate that this deficit in energy supply may explain the lack of hot coronae on A-type stars. Our results indicate an importance of nanoflares for heating and formation of the solar corona.
\end{abstract}

Keywords: stars: activity - stars: flares - stars: coronae

\section{STELLAR FLARES ACROSS HERTZSPRUNG-RUSSELL DIAGRAM}

The Sun is considered a prototype star, where the availability of high-cadence high-resolution observations together with long-term synoptic recordings allows us to study all necessary details of stellar physics. The Sun is also known for being a magnetically active star, with a variety activity phenomena connected to this activity: sunspots, faculae, prominences, and flares. It is also highly likely that the higher atmospheric layers with temperature inversion (the chromosphere and the corona) do exist thanks to the magnetic fields (Narain \& Ulmschneider 1996). Signs of magnetic activity on different stars are also known from the literature (see e.g. a recent paper by Stello et al. 2016). These signs consist of tentative evidence for existence of (star)spots (see Strassmeier 2009, for a review), prominence-like features (e.g. Collier Cameron 1996) and flares (e.g. Pettersen 1989), not to mention an evidence for activity cycles (e.g. Wilson 1978; Baliunas et al. 1995).

We will further focus on flares. Flares on the

michal@astronomie.cz
Sun seem to be a consequence of the reconnection of the entangled magnetic field above the active region (Shibata \& Magara 2011). Flares on the Sun depict various energies with smaller ones occurring frequently whereas the large flares are rare. Indications for flares were also observed on other stars, first on young or magnetically interacting stars, such as the RS CVn type, and magnetically active $\mathrm{M}$ dwarfs. Flares on Sun-like stars were not seen until the availability of the high-cadence high-precision photometry (Schaefer et al. 2000). Maehara et al. (2012) reported on observations of large-energy flares on Sun-like stars recorded in the Kepler light curves. This work was later extended by Shibayama et al. (2013) and others.

Using a similar methodology, Balona (2012) inspected over 10000 stars' light curves from Kepler public archives and discovered Sun-like flares being common not only for Sun-like stars, but also for stars of spectral types A to $\mathrm{M}$. These flares had a intensity $0.001 L_{\star}$ to $0.1 L_{\star}$, where $L_{\star}$ is a luminosity of the star, and a typical duration from minutes to several hours. The typical energy was $10^{28} \mathrm{~J}$ for the M-F stars and $10^{29} \mathrm{~J}$ for A stars. The authors also concluded that nearly all stars in question vary at a low level with a period which is likely a rotational period of the star. The flares on hotter-type stars likely do not come from the cold 
companion, because the energy of these flares is typically 100-times larger than energy of flares expected on a cool companion. This pioneering work was further elaborated later (Balona 2015), where it was found that incidence of flares on stars drops by only a factor of 4 from $\mathrm{K}-\mathrm{M}$ dwarfs to $\mathrm{A}-\mathrm{F}$ stars. This was probably largely a selection effect: contrast factor makes is easier to detect flares on cool stars, flares on hot stars need to be more energetic in order to be detected. Flare energy was strongly correlated with stellar luminosity and also correlated with stellar radius roughly as a cube function, which can be understood in terms the length of the loop undergoing reconnection being comparable to the radius of the star. The larger the star is, the larger is the active region, hence larger volume of the magnetic field (goes as a cube of the radius) and hence more energy dissipated in the flare.

The origin of the flares on A-type stars is not well understood. A standard model for Sun-like activity was developed for stars with near-surface convection zone, where the interplay between the convection, rotation, and meridional circulation redistributes and locally strengthens the magnetic field frozen in stellar plasma. One could easily apply this mechanism to stars cooler than roughly F5, where the near-surface convection zone is believed to exist and supply enough mechanical energy, which is converted to magnetism by the dynamo process. On a modelling side, first self-consistent dynamo models of other than Sun-like stars were published only recently. Augustson et al. (2013) presented a 3-D magnetohydrodynamic model for a $1.2 M_{\odot}$ rotating $\mathrm{F}$ type star, where a dynamo effect appeared in both convection and radiative zones. Magnetic field was organised into large-scale structures. It formed strong toroidal bands (wreaths) of field with intricate fibril structure and with enshrouding poloidal fields that served to link the wreaths. The portion of the radiative zone played an active role in both storing and building global-scale magnetic fields.

Stars hotter than F5 do not have an efficient mixing in near-surface layers, they only host a thin convective shell (Kallinger \& Matthews 2010) and a large convection zone in their cores. This finding is consistent with findings of Candelaresi et al. (2014), where they investigated the effects of the rotation and sunspots coverage and effective temperature on occurrence rate of superflares on G-M stars. The found that with increasing temperature the incidence rate of superflare decreases, as dynamo action gets weaker due to the decreased thickness of the sub-surface convection zone. The activity however increases with an increasing rotation rate (inverse Rossby number), flare energies are strongly correlated with rotation rate. So it would seem that without the sub-surface convection zone no near-surface dynamo process should exist. Yet, it seems that there is evidence for existence Sun-like magnetic field even on these hotter stars.

The existence of magnetic fields on A-type stars was known for some time, 5-10\% of stars in the given mass range belong to the group of chemically peculiar Ap stars with strong magnetic fields in a predominantly dipole configuration with even kilogauss strengths. These fields are considered to be fossil, as the decay time of these fields is estimated to be longer than the lifetime of the star in question (Braithwaite \& Spruit 2004). Lignières et al. (2009) reported on discovery of the magnetic field on Vega, an A star which is not an Ap star. The characteristic level of the discovered magnetic field was around $1 \mathrm{G}$ disc integrated. Further investigation (Petit et al. 2010) reveals that this magnetic field is complex with a large spot around the pole having a radial orientation of the magnetic field (with a peak intensity around $7 \mathrm{G}$ ). This polar spot is accompanied by a small number of magnetic patches in lower latitudes. Some of these small patches seem to contain also an azimuthal geometry of the magnetic field. Over two years of observations the spots seem to be quite stable. Today, there seems to be two distinct groups of A stars when the magnetic field comes in question: Ap stars with field intensity larger than $300 \mathrm{G}$ and Vega-like stars with weak 1-Gauss fields (Aurière et al. 2007; Lignières et al. 2014).

\section{STATISTICS OF STELLAR FLARES FROM KEPLER SC DATA}

From Kepler short-cadence (SC) data Balona (2012) detected altogether 3140 flare events on 209 individual stars. These flares have all the properties of solar flares, such as a rapid increase in luminosity and a much slower decrease. We aimed to study the statistical properties of the detected flares to assess the possible differences in the physics related to their origin. Therefore we divided the set of 209 stars into four corresponding spectral classes by their effective temperature, taken from the Kepler Input Catalogue. The sample is dominated by G-type stars (98 stars, 1650 flares), whereas only 12 Atype stars with 28 recorded flares are present in the sample. Balona (2012) measured flare energies $E$ and their durations $\tau$. By using a simple statistics one finds that on average, the durations of the flares do not depend on the spectral type, a typical flare duration is around 30 minutes. On the other hand, flares on A-type stars are by one order more energetic $\left(E_{\text {mean }} \approx 2 \times 10^{29} \mathrm{~J}\right)$ than on F-K stars $\left(E_{\text {mean }} \approx 3 \times 10^{28} \mathrm{~J}\right)$ and the mean power $P=E / \tau$ is also larger $\left(P_{\text {mean }}=6 \times 10^{25} \mathrm{~W}\right.$ on A stars and $P_{\text {mean }}=4 \times 10^{24} \mathrm{~W}$ on F-K stars $)$. The distribution of flare energies and mean powers is highly asymmetrical, hence mean values are not representative, however 

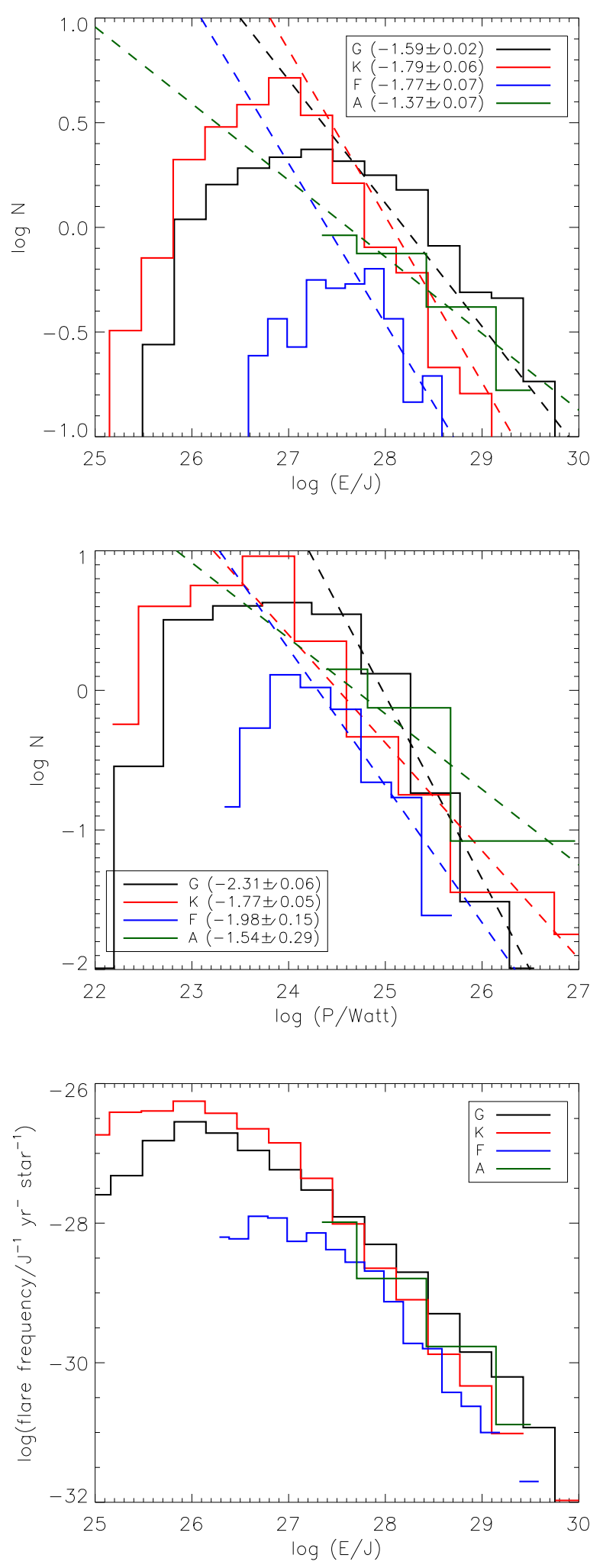

Figure 1. Upper - Histograms of flare energies for various spectral types. Estimated power-law fits according to relation (1) are over-plotted. Middle - Analogous histograms for mean flare powers. Bottom - Histograms of occurrence rates of flares, which is comparable to similar plots seen in literature (e.g. Maehara et al. 2016). for the median value similar comparative relations are found.

Studies in the past (e.g. Crosby et al. 1993; Shimizu 1995; Aschwanden et al. 2000) using various data sets have shown that on the Sun, there a variety of flares occurs differing by their total energy. Since the reconnection is essentially a process with a self-organised criticality (Aschwanden et al. 2016), the occurrence rate of solar flares in energy might be described by a power law in a form of

$$
\frac{\mathrm{d} N(E)}{\mathrm{d} E} \propto E^{\alpha},
$$

where $N(E)$ is the number of flares at a given energy $E$ and $\alpha$ is the index of the power law. For solar flares, $\alpha \sim-1.7$ based on various studies. It has been shown (e.g. Maehara et al. 2016) that flares on Sun-like G-type stars with large-energy flares (termed "superflares" in literature) also follow a similar power-law dependence with an index close to -2 .

We performed similar analysis for flares detected also on A-, F-, and K-type stars separately. The histogram of flare energies is displayed in Fig. 1 (the upper panel). The histograms for spectral types in question were normalised by number of stars in the sample and the length of Kepler SC runs. We fit the power laws to energies using the maximum likehood method (Bauke 2007) to obtain estimates for power-law coefficients, including estimates of errors. This method is considered to provide the best estimates of the power-law parameters compared to the fitting of the histogram directly, which highly depends on an arbitrary selection of the bins. The fitting was performed for the energies larger than the energy of the maximum indicated on the corresponding histogram. There is a decreasing number of flares with smaller energies, which is a selection bias due to the detection limits (Balona 2015).

The coefficients of the fitted power laws are similar for F-, and K-type stars, larger for G-type stars, and significantly larger for A-type stars. We have to point out that we cannot exclude that the less steep slope for the A stars is due to the insufficient data sample at smaller energies. The histogram for A stars does not contain decreasing branch towards lower energies, flares of energies lower than say $10^{27} \mathrm{~J}$ are below the detection limit from the light curves and we do not expect this issue to be resolved in a near future. Therefore it is not clear whether the coverage of the lowest bin in the histogram for A stars is complete. In case it is undersampled, the realistic value of the slope is steeper than estimated. The planned photometric missions such as TESS (Ricker et al. 2015) or PLATO 2.0 (Rauer et al. 2014) will not have photometric precision better than one order, which will not allow to complete the lowenergy part of flares in A stars. Both mission will po- 
tencially help with an increased statistics by observing a larger sample of stars. In the following, we assume that this effect is real and discuss possible implications.

We want to compare especially statistics of the flares on A-type stars to G-type stars, where the respective slopes are $(-1.37 \pm 0.07)$ and $(-1.59 \pm 0.02)$. These slopes that were fitted to values located on a decaying high-energy branch of the histogram (the low-energy cut-offs were $2.2 \times 10^{27} \mathrm{~J}$ and $4.2 \times 10^{27} \mathrm{~J}$ for $\mathrm{A}$ and $\mathrm{G}$ stars, respectively) are different to within $2.5 \sigma$.

Similar comparison may be performed for the histograms of the mean powers (see Fig. 1 - middle panel), where again one sees a significantly larger power-law coefficient for A-type stars than for G-type stars. For comparison with previous works in the literature (e.g. Maehara et al. 2016) we plot also a histogram of occurrence rates by energy (Fig. 1 - the bottom panel).

We performed Kolmogorov-Smirnov (K-S) tests to investigate whether the observed flare energy and mean power distribution functions differ for studied spectral types. The tests were applied to those parts of histograms, where the flare energies were larger than a chosen threshold to avoid observational biases in the low-energy part. Empirical distribution functions of A-, F-, and K-type stars were compared to a reference obtained from G-type stars. The choice of the threshold is purely arbitrary and affects the results. Therefore we performed the K-S testing for a set of threshold values. The results are summarised in Table 1 . It can be seen that when the distribution functions for energy and mean power of the flares are judged together, the histograms for A-type and G-type stars differ statistically significantly for all values of the threshold, whereas for the other spectral types when compared to G-type stars they might be considered the same especially in the region of large energies and mean powers.

\section{ARE A-TYPE STARS DIFFERENT FROM OTHER TYPES?}

To understand as to why the statistical distribution of flare energies seem to be different for A-type stars from cooler types we construct a typical stellar representative of each spectral class. We average fundamental parameters of the stars of each spectral type to form a typical star in the sample. Since the flares are in question, we would like our representatives to be rather representative of the flaring stars, hence we weight the fundamental parameters of each star contributing to the average by the number of recorded flares. This way stars flaring often are weighted more than stars with only one or few flares. The derived fundamental parameters of the representative stars is given in Table 2.

We construct stellar models for these representatives using the MESA (Paxton et al. 2011) code. By trial- and-error approach we match the following parameters of the model to those of the given representative: effective temperature $T_{\text {eff }}$, mass $M$, metalicity $Z$, and surface rotation period $P_{\text {rot }}$. For the input of the MESA code we use the model for the Sun distributed with a MESA package as a template, on the upper boundary we include a model of a grey atmosphere. In case there are multiple solutions in $T_{\text {eff }}$ (e.g. during the contraction phase and an expansion on the main sequence), we use also the average radius and $\log g$ obtained from the $\mathrm{Ke}$ pler catalogue to distinguish between these two cases.

We used models of representative stars to assess the differences in their atmospheric conditions that give us hints to explain the extended-tail in the distribution of flare energies on A-type stars compared to the other types. There are a few fundamental properties that affect the flare magnetic reconnection. In case of solar flares the magnetic reconnection sets on in the current sheets, where the electric resistivity increases suddenly from classical (collisional) to anomalous one, which is in the low solar corona (where the solar flare stars) several order of magnitudes higher than the collisional one. This anomalous resistivity occurs when the drift velocity of electrons carrying the electric current overcomes some critical velocity (usually ion-acoustic speed or thermal electron speed - Norman \& Smith 1978, Priest 1981). Similar start of the magnetic reconnection can be expected in all types of stars with hot corona. Although the initial conditions for the magnetic reconnection in A-type stars are probably different (due to the missing hot X-ray corona), very soon after the start of the magnetic reconnection the temperature in the reconnection site strongly increases. Thus, in the following times the magnetic reconnection and its spreading into larger volume is in the hot plasma regime.

It is not possible to properly (i.e. including the possible temperature inversion layers, such as chromosphere or corona, such as the set of VAL, Vernazza et al. 1981, models in the case of the Sun) model the stellar atmospheres of the representative stars, hence we stick to the estimates from the grey atmospheric models computed for optical depths $10^{-4}$ and larger. We analyzed all plasma parameters of these atmospheres. The most distinct difference was found in plasma densities. The density on A-star is more than one order lower than on cooler stars (see Fig. 2).

Now a question arises what is an effect of lower density at flare site. Firstly, the radiative losses are much lower, because they are proportional to the plasma density to the square (Priest 1981). Thus, owing to this reduction of energy losses higher temperatures in flares can be reached. Secondly, the threshold for the currentdriven instabilities generating the anomalous resistivity decreases with the density decrease. Namely, a drift 
Table 1. Effect of the chosen threshold on descriptive statistics of the flares. For various threshold values the probabilities from Kolmogorov-Smirnof tests (always in pairs for both the histograms of energy and histograms of mean powers) are given. By a star we indicate situation, where with lesser than $5 \%$ probability we make an error by assuming that the distribution function of both tested samples are different for both the energies and mean powers.

\begin{tabular}{r|ccc} 
Threshold [J] & K-S A-G & K-S F-G & K-S K-G \\
\hline $2 \times 10^{27}$ & $\star 0.02,3 \times 10^{-5}$ & $\star 7 \times 10^{-4}, 1 \times 10^{-3}$ & $\star 2 \times 10^{-19}, 7 \times 10^{-40}$ \\
$4 \times 10^{27}$ & $\star 0.12,4 \times 10^{-3}$ & $\star 5 \times 10^{-4}, 7 \times 10^{-3}$ & $\star 8 \times 10^{-7}, 4 \times 10^{-9}$ \\
$6 \times 10^{27}$ & $\star 0.19,6 \times 10^{-3}$ & $2 \times 10^{-5}, 0.07$ & $\star 0.02,0.05$ \\
$8 \times 10^{27}$ & $\star 0.23,4 \times 10^{-3}$ & $3 \times 10^{-3}, 0.11$ & $0.08,0.29$ \\
$1 \times 10^{28}$ & $\star 0.03,5 \times 10^{-3}$ & $0.04,0.83$ & $0.16,0.12$ \\
$1.2 \times 10^{28}$ & $\star 0.04,1 \times 10^{-3}$ & $0.11,0.78$ & $0.15,0.03$ \\
$1.4 \times 10^{28}$ & $\star 0.05,2 \times 10^{-3}$ & $0.42,0.57$ & $0.13,0.05$
\end{tabular}

Table 2. Fundamental parameters of typical representatives of stars for each spectral type considered. In the first part are those derived from our sample of stars, in the second part the parameters are derived from the model

\begin{tabular}{l|ccccc|cc}
\hline Sp. type & $T_{\text {eff }}[\mathrm{K}]$ & $M\left[M_{\odot}\right]$ & $R\left[R_{\odot}\right]$ & $Z$ & $P_{\text {rot }}[$ days $]$ & $R_{\text {MESA }}\left[R_{\odot}\right]$ & $v_{\text {rot }}\left[\mathrm{km} \mathrm{s}^{-1}\right]$ \\
\hline A & 8570 & 2.29 & 3.28 & 0.017 & 5.54 & 3.42 & 31.2 \\
F & 6360 & 1.41 & 2.38 & 0.014 & 2.59 & 2.33 & 46.8 \\
$\mathrm{G}$ & 5490 & 1.00 & 1.51 & 0.014 & 4.05 & 1.74 & 22.0 \\
$\mathrm{~K}$ & 4200 & 0.61 & 0.76 & 0.025 & 5.68 & 0.66 & 5.9 \\
\hline
\end{tabular}

velocity of electrons $v_{\mathrm{D}}$ carrying the electric current is inversely proportional to the electron density $n_{\mathrm{e}}$

$$
v_{\mathrm{D}}=j /\left(e n_{\mathrm{e}}\right)
$$

where $j$ is the electric current density and $e$ the electron charge. It means that the anomalous resistivity can be generated in the current sheet with smaller derivative of the magnetic field across the current sheet as follows

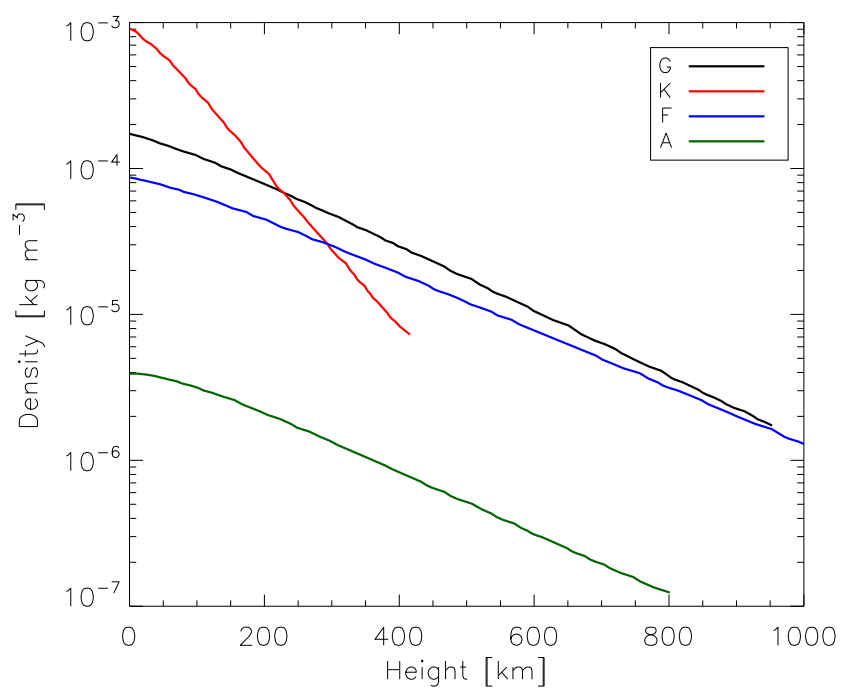

Figure 2. Density profiles in the atmospheres of representative stars from MESA models. Optical depth was converted to height above level of unity optical depth using the model of a grey atmosphere. from

$$
j \propto \frac{\partial B}{\partial x}
$$

where $B$ is the magnetic field component along the current sheet and $x$ is the coordinate across the current sheet. Namely, in the current sheet the only non-zero derivative of the magnetic field is that across the current sheet.

A-type stars do not have sub-surface convection zones, however observations indicate that they must posses photospheric magnetic fields of solar type, hence with a mixed polarity. This magnetic field probably originates from the dynamo process occurring in the convective core. Numerical simulations (Featherstone et al. 2011) claim to see a significant dynamo action resulting even in super-equipartition magnetic fields in the interior of not only A-type stars, but also on B-type stars (Augustson et al. 2016). A super-equipartition (with respect to the convective motion) field may become unstable, buoyant, and rise through a radiative envelope towards stellar photosphere. There are no significant bulk motions in this radiative envelope, hence the field rises up basically in form of the loops and is not dispersed by convective motions. Since the gas density decreases as does gas pressure, the field fans out in order to maintain the pressure equilibrium: It becomes spatially larger, however with smaller intensity. It is analogous to the loops in the solar atmosphere, fanning out from active regions. Therefore we may expect the appearance of the magnetic fields with mixed polarity that have a larger extent.

The observational evidence for large spots on A stars is 
problematic at least. Balona (2013) estimated the sizes of spots responsible for the rotational modulation of Astar light curves and found that these spots are comparable in size with those on the Sun. This contradicts not only our expectations formulated above, but also findings with regards to G-type stars (Maehara et al. 2016), where large spots (covering 1\% of stellar surface and more) are a necessary condition for superflares to ignite.

It is possible that the spot areas measured by Balona (2013) are severely underestimated from three reasons: First, the methodology assumes that the light-curve variations are due to a dark round spot. Then the fractional decrease in bolometric flux is proportional to the fractional area of the spot with respect to the visible hemisphere of the star. In reality, these spots are never completely dark, the relative contrast of sunspots is larger for sunspots with a stronger magnetic field. We assumed above that the intensity of the magnetic field in the photosphere of A stars is lower ( 100 G) than on the Sun $(\sim 1000 \mathrm{G})$, hence one may safely assume that the relative contrast of spots and the surrounding photosphere be also lower. In effect, this leads to an underestimate of size of the spot. Second, the method is not sensitive to spots that remain on a visible hemisphere of the star, e.g. to spots in larger latitudes of star with inclined axis of rotation. And third, Cantiello \& Braithwaite (2011) pointed out a possibility of a weak dynamo action even in near-surface convective shells of early-type stars. Such a dynamo would result in brighter spots, similarly to faculae on the Sun. These bright spots would compensate for some of the darkening caused by dark spots in the light-curve modulated by rotation and again lead to underestimation of the spot size.

Numerical simulations (e.g. Beeck et al. 2013, 2015) suggest that a typical scale of convection increases with increasing surface temperature from around $1 \mathrm{Mm}$ for Sun-like stars to $\sim 5 \mathrm{Mm}$ for a F3 star and $\sim 15 \mathrm{Mm}$ for an A star (Freytag et al. 2012). The magnetic flux is dispersed to the same (and larger) scales, which provides an indirect hint for large-scale magnetic fields to exist in photospheres of A stars.

Thanks to the decreased profile of electron density compared to the $\mathrm{F}-\mathrm{K}$ stars the flare may ignite in regions closer to the photosphere of the A star, where we assume that the intensity of the magnetic field is $\sim 100 \mathrm{G}$ (which is comparable to the magnetic field intensity at the reconnection site of solar flares). The flare will then engulf a large volume of the magnetic field resulting in a flare with a large energy released in total. Since the incidence of flares per star is lower for A-type stars than for cooler types (there is on average 2.33 flares per star for A-type, 4.20 for F-type, 16.83 for G-type, and 22.34 for K-type) is would also seem that it takes a larger period of time for the dynamo to create such a large magnetic field. Theoretical considerations for the case of our Sun (e.g. Shibata et al. 2013) also show that the occurrence rate of large solar flares is limited by the finite efficiency of the dynamo process.

\section{4. (NON-)MAGNETIC A-TYPE STARS}

There is a tentative evidence for Sun-like flares occurring also on A-type stars. The Sun-like flares need complex topologies of active regions. There is no observational evidence for existence of such magnetic fields in atmospheres of A stars. A well known group of A stars does posses observed magnetic fields. Ap stars are peculiar stars of type A which show overabundances of some rare metals. They also have strong magnetic fields typically reaching up to tens of $\mathrm{kG}$. In most cases a field which is dipole-like and is not aligned with a rotation axis. It is generally assumed that the the magnetic field of Ap stars is the fossil in origin, in which the field is a relic of the initial field in the interstellar medium. The estimated diffusion time for such a magnetic field in the conditions in the interior of a typical A star is $\sim 10^{9}$ years (Braithwaite \& Spruit 2004).

None of the flaring A stars in our sample belongs to the group of Ap stars. It is not surprising. We have mentioned a generally accepted hypothesis that in order to ignite a Sun-like flare a region with high current density is needed to exist. The bipolar global magnetic field does not provide conditions for such high-currentdensity regions to exist. Therefore one should not expect a typical Ap star to flare.

On the other hand, Featherstone et al. (2011) performed a fully 3-D magnetohydrodynamic modelling of a $2 M_{\odot}$ A star with included fossil magnetic field having a twisted toroidal shape with a strong dipolar component. The dynamo takes place in the convective core which builds up a strong mean field and with excursions towards super-equipartition intensities with respect to the convection. The authors speculated that these fields might possibly become buoyant (due to the diffusive limitations in the code such a rise was not observed during various runs), ultimately contributing to form large spots (Featherstone 2011). Due to the feedback of the magnetic field on convection, there is almost no latitudinal differential rotation in the core of such a star. The results of this simulation interestingly fit to a scenario by Lignières et al. (2014). The authors note that there is a magnetic gap between A stars with measured large-scale magnetic fields stronger than a few hundreds Gauss (around 5-10\% of A stars) and A-stars of Vegatype, where only weak $(\sim 1 \mathrm{G})$ magnetic fields were measured spectroscopically. The authors point out that if a magnetic field is stronger than some $300 \mathrm{G}$, it is strong 
enough to avoid macroscopic mixing through differential rotation, thermal convection, or stellar winds, and the star becomes a Ap star with chemical peculiarity. Weaker fields do not prevent the mixing and cannot prevent their winding-up by differential rotation into strong toroidal fields. Such configuration of the magnetic field is expected to become unstable (e.g. a Tayler instability, Tayler 1973), which transforms the initially largescale field configuration into a new configuration with mixed polarities at the length scale of the instability. On the contrary, if the initial magnetic field is strong enough, Maxwell stresses impose uniform rotation and eventually lead to a stable configuration. The critical field intensity discriminating the two different outputs of coupling between plasma streaming and magnetic field inside the A star is around $300 \mathrm{G}$. This value was experimentally established earlier by Aurière et al. (2007), who studied spectroscopically a sample of $28 \mathrm{Ap} / \mathrm{Bp}$ stars. They inferred the intensity of the dipole magnetic field and found that its histogram shows a peak around $1000 \mathrm{G}$ falling off to both smaller and larger fields. There were no stars with dipole field strength less than $300 \mathrm{G}$. The authors claim that their results is neither detection threshold effect nor selection effect.

We speculate that flaring A stars underwent Tayler instability in the past, which transformed their initially dipole fossil fields into organised toroidal ones. These localised fields possibly contain patches of both polarities, as solar active regions do. Therefore, they do not contribute to a polarimetric signal, because the discintegrated polarimetric signals largely cancel out. The localised magnetic fields in photospheres of such stars would not be measurable using standard spectroscopic methods.

\section{IMPLICATIONS FOR CORONAL HEATING}

The presence of stellar coronae is traditionally connected with the radiation in the band of X-rays, due to the expected high temperature of a few million degrees. X-ray fluxes were studied in several survey projects, namely in ROSAT all-sky survey. Several authors used these measurements to cross-identify the X-ray sources with e.g. stellar catalogues. It was found (Huensch et al. 1998; Schröder \& Schmitt 2007) that the ratio of stars with detected X-rays and all stars of the given spectral type is not uniform among spectral types, e.g. there is a prominent underabundance of X-ray-active stars between spectral types B3 and F0 (see Fig. 2 of Schröder \& Schmitt 2007). On the high-temperature wall of this 'hole' the indications of Xrays coming from instabilities in the radiatively driven winds on early-B and hotter stars are visible, on the low-temperature wall the X-rays increase due to the expected onset of $\alpha \Omega$ dynamo on $\mathrm{F}$ stars, related to the onset of near-surface convection zone. The incidence of $\mathrm{X}$-rays among A-type stars is less than $20 \%$, which may be interpreted as non-existence of hot stellar coronae around A-stars.

The different statistics of occurrence rates of flares on A-type stars compared to cooler types provides hints to speculate about the corona formation. The existence of the solar corona has not been successfully explained since almost 75 years (De Moortel \& Browning 2015). One class of models of coronal heating assumes that flares play a significant role (Parker 1988). Energetic considerations convincingly show that for the Sun, the energy released by large solar flares is not sufficient to cover radiative losses from the 1-million degree corona. There is another evidence that the heating should be more-or-less stationary, which again precludes large flares occurring at most once a day to play a significant role. It is therefore assumed that the role of heating events is played by small-scale flares occurring more frequently, in general termed nanoflares with energies around $10^{17} \mathrm{~J}$. If the index of the power law in relation (1) is -2 or smaller, then the total energy released by flares is dominated by low-energy events. For the Sun the power-law index is close to -2 .

From our results it seems that the power-law index is similar also for $\mathrm{F}$-, and $\mathrm{K}$-type stars, hence the existence of the hot corona around these stars may be justified due to the small flares occurring there. However, for the A-type stars, the power-law is not that steep, around -1.4 , hence the energy budget released by flares is dominated by large-energy events. By extrapolating the fits in the histograms in Fig. 1 towards low-energy part and integrating the spectrum from nanoflare energies $10^{17} \mathrm{~J}$ to superflare energies $10^{30} \mathrm{~J}$ we obtain estimates for energy flux into the higher atmosphere by eruptive events. We found that the total integrated flux (with $1 \sigma$ bounds) is $(6.7-6.8) \times 10^{21} \mathrm{~W}$ for the representative G-type star, $(3.9-5.6) \times 10^{21}$ for the K-type, and $(1.4-1.6) \times 10^{21}$ for the F-type star. Even though these number are very rough estimates, they are not far from e.g. a quiet-Sun X-ray luminosity of around $5 \times 10^{20}$ (Vaiana \& Rosner 1978) measured for the Sun. For the representative A-star the energy flux by flare events using the same methodology is $(6.2-7.3) \times 10^{21} \mathrm{~W}$. Taking into account that these stars have a larger surface area, the energy flux density into higher atmosphere is 4- to 5 -times larger in case of the representative G-type star than in case of the A-type star.

There are two noticeable issues. First, the slope determined for G-type stars is also significantly lower than critical value of -2 , distinguishing the dominance of the contribution by small-energy flares and large-energy events. It is evident in the upper panel of Fig. 1 that the shape of the histogram for G stars around the loca- 
tion of the maximum occurrence rate is different from histograms for $\mathrm{K}$ and $\mathrm{F}$ stars. The width of the maximum of the G-star histogram is much larger than in the other two cases. This fact together with the automatic selection of the low-energy cut-off at the position of the histogram maximum leads to a shallower slope of the fitted power law. Increasing the low-energy cut-off to e.g. $10^{28} \mathrm{~J}$ leads to a derived slope of $(-1.70 \pm 0.03)$ and the extrapolated total flux by flare events $(7.0-7.8) \times 10^{21} \mathrm{~J}$. The energy density would then be 5 - to 6-times larger than for the A stars. The reason for existence of a pseudo-plateau in the histogram of flares in G stars is unknown and deserves a future investigation.

The second issue is that our estimates are based on observations of superflare stars, whereas our Sun does have a hot corona and it does not seem to be a superflare stars. There are indications that the lack of superflares on our Sun may simply be a consequence of short observational series available for analysis. Shibata et al. (2013) showed that the occurence rates for superflares on Kepler G-type stars group in the occurence rate diagram around the same power law as do the occurence rates for solar flares of a large range of energies from nanoflares to regular solar flares (see their Figure 1). Simplistic estimates made by the authors also do not exclude possibility of superflares occuring on our Sun, e.g. $10^{28} \mathrm{~J}$ flare could on average happen once in 5000 years.

It has to be noted that our speculative claims are based on extrapolation and a silent assumption that the occurrence rate of flares follows the same power law over many order of magnitude. With a current instrumentation it is not possible to verify our ideas directly by observations. However, there are many pieces of a puzzle described above that seem to fit together to draw a physically sound picture of the origin of the flares and hot corona formation. These pieces of evidence include both observations and state-of-the-art numerical simulation, thereby being largely independent.

The apparently lower energy flux by flaring events may easily explain the lack for evidence for the hot X-ray emitting coronae on A-type stars and strengthen the role of nanoflares in heating the corona of the Sun.

\section{SUMMARY}

In agreement with previous studies, we assume that in the A-type flare stars the magnetic field is amplified by dynamo processes in the convective core of the stars. Then part of the generated magnetic field becomes unstable, buoyant and rises as magnetic ropes through a radiative envelope towards stellar photosphere. During this process the magnetic ropes become larger and their magnetic field decreases. Based on the flare statistics we propose that this process generates more large-scale structures and less small-scale structures of the magnetic field than in the case of Sun-like stars. Owing to the relative shortage of the small-scale structures, a number of small flares (nanoflares) is relatively small and the upper atmospheric layers of the A-like flare star is not sufficiently heated and thus a hot X-ray emitting corona cannot be formed. On the other hand, the large-scale structures of the magnetic field in large volumes can be dissipated in huge flares.

By modelling the stellar atmosphere we show that the gas density in the atmospheres of A stars is lower, hence the radiative losses are also much lower. Owing to this reduction of energy losses higher temperatures in flares can be reached. The lower densities in the photosphere and in the above laying layers in the A-type flare stars comparing to cooler stars, the threshold for generation of the anomalous resistivity, which is important in any flares, can be reached for smaller magnetic field derivatives in flare current sheets.

Contrary to the A-type flare stars, in the Sun and cooler stars the hot corona is formed. For cooler stars the power-law index of the flare occurrence looks to be steeper than that for the A-type flare stars, which shows relatively more nanoflares in these stars. Moreover, it indicates an importance of nanoflares for heating and formation of the solar corona.

We are very grateful to Luis Balona (South African Astronomical Observatory) for making the measurements of stellar flares public through VizieR interface. The authors were supported by the institute research project RVO:67985815 to Astronomical Institute of Czech Academy of Sciences. M. ̌́. further acknowledges a support from the grant 15-02112S, M.K. from grant P209/12/0103, both grants were awarded by the Czech Science Foundation. The original data are based on observations collected by the Kepler mission. Funding for the Kepler mission is provided by the NASA Science Mission directorate. These observations were obtained from the Mikulski Archive for Space Telescopes (MAST). STScI is operated by the Association of Universities for Research in Astronomy, Inc., under NASA contract NAS5-26555. Support for MAST for non-HST data is provided by the NASA Office of Space Science via grant NNX09AF08G and by other grants and contracts." We thank Sacha Brun for useful advices. We thank the anonymous referee for useful comments that improved the paper. 
Aschwanden, M. J., Tarbell, T. D., Nightingale, R. W., et al. 2000, ApJ, 535, 1047

Aschwanden, M. J., Crosby, N. B., Dimitropoulou, M., et al. 2016, SSRv, 198, 47

Augustson, K. C., Brun, A. S., \& Toomre, J. 2013, ApJ, 777, 153

—. 2016, ArXiv e-prints, arXiv:1603.03659

Aurière, M., Wade, G. A., Silvester, J., et al. 2007, A\&A, 475, 1053

Baliunas, S. L., Donahue, R. A., Soon, W. H., et al. 1995, ApJ, 438, 269

Balona, L. A. 2012, MNRAS, 423, 3420

-. 2013, MNRAS, 431, 2240

-. 2015, MNRAS, 447, 2714

Bauke, H. 2007, European Physical Journal B, 58, 167

Beeck, B., Cameron, R. H., Reiners, A., \& Schüssler, M. 2013, A\&A, 558, A49

Beeck, B., Schüssler, M., Cameron, R. H., \& Reiners, A. 2015, A\&A, 581, A42

Braithwaite, J., \& Spruit, H. C. 2004, Nature, 431, 819

Candelaresi, S., Hillier, A., Maehara, H., Brandenburg, A., \& Shibata, K. 2014, ApJ, 792, 67

Cantiello, M., \& Braithwaite, J. 2011, A\&A, 534, A140

Collier Cameron, A. 1996, in IAU Symposium, Vol. 176, Stellar Surface Structure, ed. K. G. Strassmeier \& J. L. Linsky, 449

Crosby, N. B., Aschwanden, M. J., \& Dennis, B. R. 1993, SoPh, 143,275

De Moortel, I., \& Browning, P. 2015, Philosophical Transactions of the Royal Society of London Series A, 373, 20140269

Featherstone, N. A. 2011, PhD thesis, University of Colorado at Boulder

Featherstone, N. A., Browning, M. K., Brun, A. S., \& Toomre, J. 2011, Journal of Physics Conference Series, 271, 012068

Freytag, B., Steffen, M., Ludwig, H.-G., et al. 2012, Journal of Computational Physics, 231, 919

Huensch, M., Schmitt, J. H. M. M., \& Voges, W. 1998, A\&AS, 132,155

Kallinger, T., \& Matthews, J. M. 2010, ApJL, 711, L35

Lignières, F., Petit, P., Aurière, M., Wade, G. A., \& Böhm, T. 2014, in IAU Symposium, Vol. 302, Magnetic Fields throughout Stellar Evolution, ed. P. Petit, M. Jardine, \& H. C. Spruit, 338-347
Lignières, F., Petit, P., Böhm, T., \& Aurière, M. 2009, A\&A, 500, L41

Maehara, H., Shibayama, T., Notsu, Y., et al. 2016, ArXiv e-prints, arXiv:1601.01132

Maehara, H., Shibayama, T., Notsu, S., et al. 2012, Nature, 485, 478

Narain, U., \& Ulmschneider, P. 1996, SSRv, 75, 453

Norman, C. A., \& Smith, R. A. 1978, A\&A, 68, 145

Parker, E. N. 1988, ApJ, 330, 474

Paxton, B., Bildsten, L., Dotter, A., et al. 2011, ApJS, 192, 3

Petit, P., Lignières, F., Wade, G. A., et al. 2010, A\&A, 523, A41

Pettersen, B. R. 1989, SoPh, 121, 299

Priest, E. R. 1981, Solar flare magnetohydrodynamics

Rauer, H., Catala, C., Aerts, C., et al. 2014, Experimental Astronomy, 38, 249

Ricker, G. R., Winn, J. N., Vanderspek, R., et al. 2015, Journal of Astronomical Telescopes, Instruments, and Systems, 1, 014003

Schaefer, B. E., King, J. R., \& Deliyannis, C. P. 2000, ApJ, 529, 1026

Schröder, C., \& Schmitt, J. H. M. M. 2007, A\&A, 475, 677

Shibata, K., \& Magara, T. 2011, Living Reviews in Solar Physics, 8, doi:10.12942/lrsp-2011-6

Shibata, K., Isobe, H., Hillier, A., et al. 2013, PASJ, 65, arXiv:1212.1361

Shibayama, T., Maehara, H., Notsu, S., et al. 2013, ApJS, 209, 5 Shimizu, T. 1995, PASJ, 47, 251

Stello, D., Cantiello, M., Fuller, J., et al. 2016, Nature, 529, 364

Strassmeier, K. G. 2009, A\&A Rv, 17, 251

Tayler, R. J. 1973, MNRAS, 161, 365

Vaiana, G. S., \& Rosner, R. 1978, ARA\&A, 16, 393

Vernazza, J. E., Avrett, E. H., \& Loeser, R. 1981, ApJS, 45, 635 Wilson, O. C. 1978, ApJ, 226, 379 